\title{
The Kinesin Superfamily Handbook
}

\section{Transporter, Creator, Destroyer}

Edited by

Claire T. Friel

First edition published 2020

\section{The Kinesin-15 Family Long-Distance Transporters}

Hauke Drechsler, Jaspreet Singh Grewal and Andrew D. McAinsh

CC BY-NC-ND 4.0
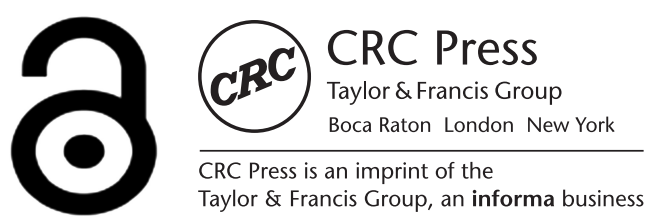


\title{
11 The Kinesin-15 Family
}

\author{
Hauke Drechsler, Jaspreet Singh Grewal \\ and Andrew D. McAinsh
}

\section{CONTENTS}

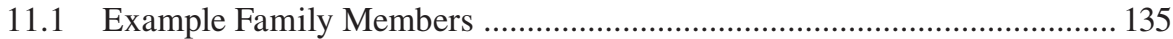

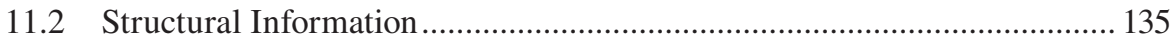

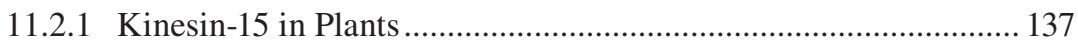

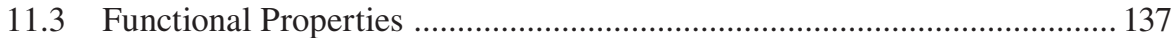

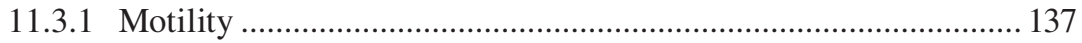

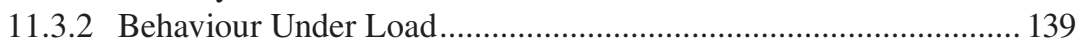

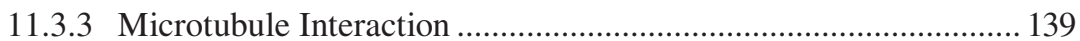

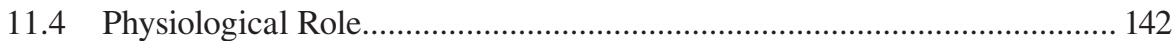

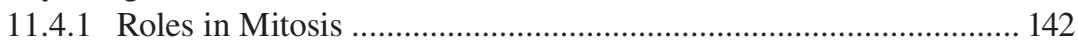

11.4.2 Establishing the Cortical Division Zone and Phragmoplast

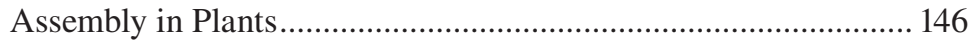

11.4.3 Postmitotic Functions in Neuronal Development ......................... 147

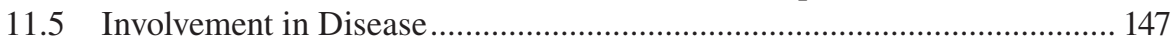

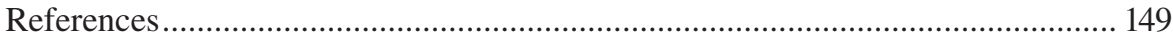

Kinesin-15s are a family of processive, plus-end-directed motors that can function as either homodimers or homotetramers. They can cross-link and bundle microtubules and play crucial roles in mitosis.

\subsection{EXAMPLE FAMILY MEMBERS}

Mammalian: KIF15 (KLP2)

Caenorhabditis elegans: KLP-18

Xenopus laevis: KLP2

Arabidopsis thaliana: POK1, POK2, PAKRP1

Physcomitrella patens: KINID1a, KINID1b

\subsection{STRUCTURAL INFORMATION}

Animal Kinesin-15s typically consist of a heavy chain containing 1400 amino acids with a molecular weight of $160 \mathrm{kDa}$ (Boleti, Karsenti, and Vernos 1996, Sueishi, Takagi, and Yoneda 2000, Rogers et al. 2000, Buster et al. 2003). No further 
regulatory or accessory medium or light chains are required for motility (Drechsler et al. 2014, Drechsler and McAinsh 2016, Sturgill et al. 2014, Reinemann et al. 2017, Milic et al. 2018). The Kinesin-15 heavy chain features a short N-terminal extension (aa1-19 in human KIF15, hKIF15 from here on) preceding the N-terminal motor domain including the neck-linker (aa19-375 in hKIF15, crystal structure available at PDB ID: 4bn2 or in Klejnot et al. 2014), which is followed by a long alpha-helical stalk forming an interrupted coiled-coil (aa376-1148 in hKIF15) and finally by the C-terminal tail domain (aa1149-1388 in hKIF15) (Boleti, Karsenti, and Vernos 1996, Klejnot et al. 2014, Buster et al. 2003) (Figure 11.1). The function of the N-terminal extension is currently unknown. However, similar N-terminal extensions are involved in cargo binding (Kinesin-3 (Gruneberg et al. 2006) and Kinesin-7 (Drechsler, Tan, and Liakopoulos 2015, Roberts, Goodman, and ReckPeterson 2014)), force generation (Kinesin-1 (Khalil et al. 2008, Hwang, Lang, and Karplus 2008)) or microtubule association (Kinesin-5 (Stock, Chu, and Hackney 2003, Britto et al. 2016) and Kinesin-7 (Drechsler, Tan, and Liakopoulos 2015)).

The simplest oligomerisation state for Kinesin-15 motors is a homodimer: both Xenopus laevis and Strongylocentrotus purpuratus (sea urchin) Kinesin-15, partially purified from meiotic egg extracts, are dimeric with a measured Stokes radius of $\sim 10 \mathrm{~nm}$ and a sedimentation coefficient of $\sim 8 \mathrm{~S}$, consistent with being an elongated molecule with a native molecular weight of $334 \mathrm{kDa}$ (Rogers et al. 2000, Wittmann et al. 1998). In contrast, the human Kinesin-15 (hKIF15) can also assemble into homotetramers (i.e., a dimer-of-dimers) both in vivo and in vitro (Mann, Balchand, and Wadsworth 2017, Drechsler et al. 2014, Drechsler and McAinsh 2016, Sturgill et al. 2014). The tetrameric and dimeric forms of hKIF15 co-exist in a saltdependent equilibrium. Regardless of its source (i.e., recombinant or endogenous (Drechsler and McAinsh 2016)), hKIF15 is primarily tetrameric ( 12 S) at low to physiological ionic strength ( $\mathrm{I}=75 \mathrm{mM}$ to $225 \mathrm{mM})$, and primarily dimeric $(\sim 8 \mathrm{~S})$ at an ionic strength above $300 \mathrm{mM}$ (Drechsler and McAinsh 2016, Drechsler et al. 2014, Sturgill et al. 2014, Mann, Balchand, and Wadsworth 2017). It is important to note that the dimeric form of hKIF15 - purified at high ionic strength - appears to be auto-inhibited (Sturgill et al. 2014), whereas KIF15 tetramers are constitutively active (Drechsler and McAinsh 2016, Drechsler et al. 2014, Mann, Balchand, and Wadsworth 2017). As a result, experiments with the dimeric form are only possible if the motor is either truncated (i.e., aa1-700 (Sturgill et al. 2014, Milic et al. 2018)) or by releasing autoinhibition with an antibody targeting the C-terminus of hKIF15 (Sturgill et al. 2014). There is currently no structural data on how the two hKIF15 dimers arrange to form the tetramer, although mass spectrometry cross-linking experiments suggest a parallel tetramer, with all motor domains gathered at one end of the tetramer (Hussain, McAinsh and Jones, unpublished). This arrangement would be distinct from the arrangement of heavy chains in the - also tetrameric Kinesin-5, which has overlapping functions with KIF15 (see below). In Kinesin-5s, two antiparallel coiled-coils form a four-helical bipolar assembly (BASS) domain (Acar et al. 2013, Scholey et al. 2014), resulting in a dumbbell structure and exposing a motor domain pair at each end of the rod-like tetramer (see Chapter 6 for details). 


\subsubsection{Kinesin-15 in Plants}

In contrast to their orthologues in animals, plant Kinesin-15s are poorly characterised at the molecular level. Currently, we know two different species of plant Kinesin-15 that are either slightly shorter (i.e., KINID1a/b: 1200 amino acids, and PAKRP1/1L: 1300 amino acids) or considerably longer (i.e., POK1: 2066 amino acids, and POK2: 2771 amino acids) than their animal counterparts (Figure 11.1). Studies on a truncated POK2 variant, POK $2_{1-589}$, suggest that no additional medium or light chains are required for plant Kinesin-15 motility in vitro and that dimers are the minimal functional unit (Chugh et al. 2018). Two further structural features distinguish plant Kinesin-15s: (i) the relative low abundance of extended coiled-coil stretches in their stalks (particularly in PAKRP1/1L and KINID1a/b) and (ii) the remarkably long $\mathrm{N}$-terminal extension preceding the respective motor domains, ranging from 39 amino acids in KINID1b to the enormous 190 amino acids in POK2 (Figure 11.1). In the case of POK2, this N-terminal extension is required for the processive movement (Chugh et al. 2018), with conflicting reports as to whether there is a second ATP-independent microtubule-binding site (Chugh et al. 2018, Herrmann et al. 2018). The C-terminus of AtPOK1 (aa1683-2066) binds to the preprophase band (PPB) resident MAP TAN ("tangled") (Lipka et al. 2014), while the N-terminus (aa1-189) and C-terminus (aa2083-2771) of AtPOK2 interact with the plant PRC1/ ASE1 orthologue MAP65-3, thereby restricting AtPOK2 localisation to the plant's phragmoplast midzone (Herrmann et al. 2018) (Figure 11.1).

\subsection{FUNCTIONAL PROPERTIES}

\subsubsection{MotILITY}

The human KIF15 is a processive plus-end-directed motor, moving in vitro at 130-200 $\mathrm{nm} \mathrm{s}^{-1}$ without load (Drechsler et al. 2014, Sturgill et al. 2014, Mann, Balchand, and Wadsworth 2017, McHugh et al. 2018). Under certain conditions, i.e., by increasing the assay temperature or the ionic strength, unloaded velocities have been reported to reach $\sim 500 \mathrm{~nm} \mathrm{~s}^{-1}$ (Drechsler and McAinsh 2016, Milic et al. 2018). Processive runs can be interrupted by prolonged pauses ( $\sim \mathrm{s}$ (Drechsler et al. 2014)) and the hKIF15 motor is capable of switching between processive and diffusive modes of movement (Drechsler et al. 2014). Single motors dwell at microtubule plus ends for $20 \mathrm{~s}$ (Drechsler et al. 2014), leading to motor accumulation (Drechsler and McAinsh 2016). At microtubule intersections, hKIF15 motors can switch microtubule tracks with a likelihood of one in five, allowing the motor to navigate long distances through complex microtubule networks (Drechsler et al. 2014, Mann, Balchand, and Wadsworth 2017). Interestingly, short episodes of processive minus-end-directed movement have been detected, although this is infrequent and restricted to shorter run length and residency time compared to plus-end-directed motors (Drechsler et al. 2014, Mann, Balchand, and Wadsworth 2017). The oligomerisation state does not appear to affect the motor's velocity $\left(\sim 140 \mathrm{~nm} \mathrm{~s}^{-1}\right.$ for tetramers vs. $\sim 190 \mathrm{~nm} \mathrm{~s}^{-1}$ for dimers that are activated by an antibody bound to the C-terminus). However, activated dimers exhibit a reduced run length $(\sim 0.6 \mu \mathrm{m}$ vs. 


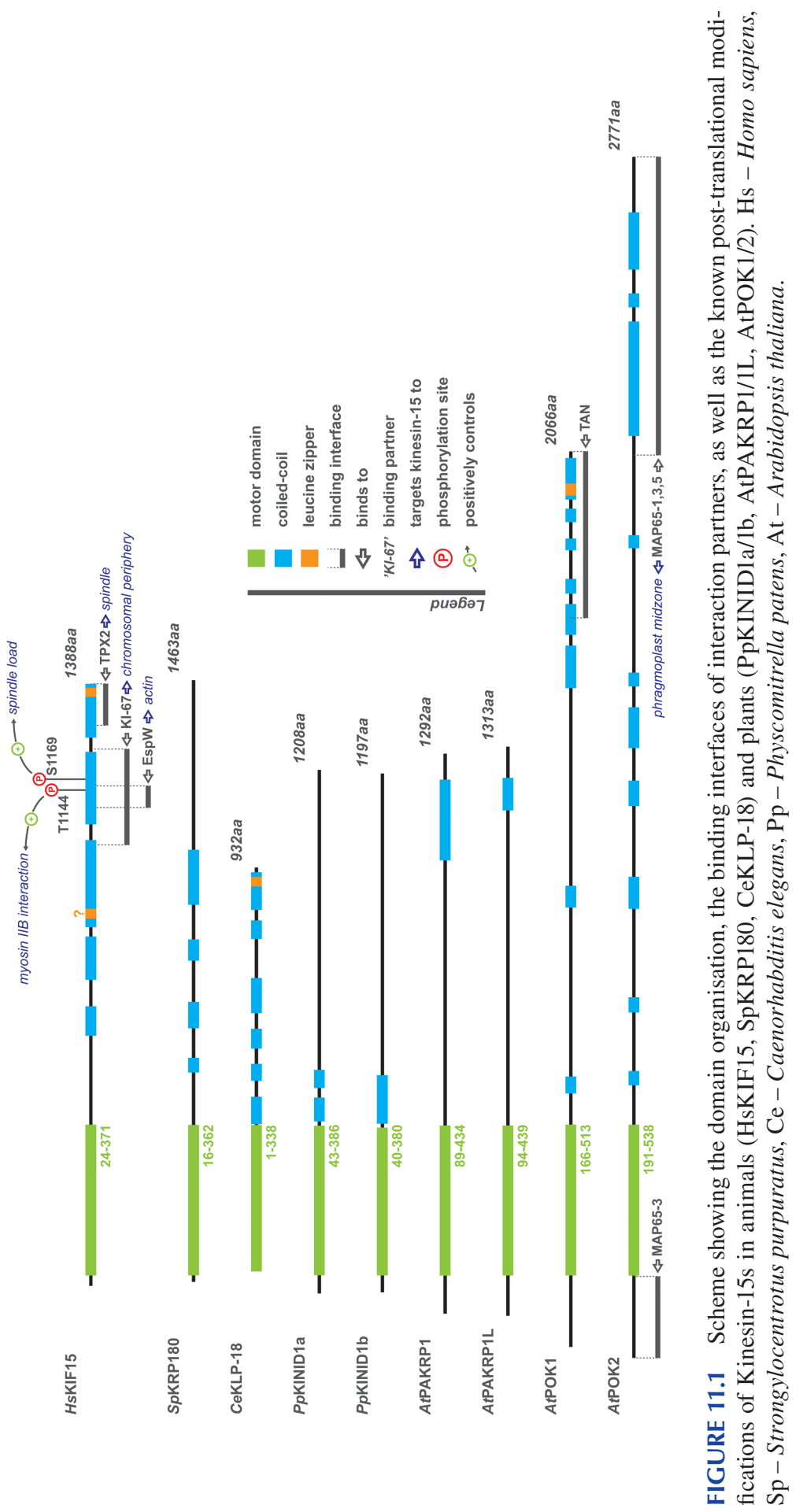


$\sim 2 \mu \mathrm{m}$ for tetramers) and residency time ( $\sim \mathrm{s}$ vs. $\sim 26 \mathrm{~s}$ for tetramers) (Drechsler et al. 2014, Sturgill et al. 2014). This difference might be explained by the presence of a second pair of motor domains in the tetramer, which increases the microtubule affinity at the expense of velocity. However, the hKIF15 constructs used by Sturgill et al. (2014) lack two-thirds of the N-terminal extension, which may affect motor performance (see Structural Information Section 11.2).

\subsubsection{Behaviour Under Load}

During processive runs, hKIF15 motors have been reported to stall at $\sim 3 \mathrm{pN}$ (Reinemann et al. 2017, Drechsler et al. 2014) or at $\sim 6 \mathrm{pN}$ (McHugh et al. 2018, Milic et al. 2018), differences that likely depend on the construct and assay method used. External motor load strongly influences hKIF15 performance, as its velocity scales inversely with the amount of hindering forces (i.e., forces directed against the walking direction) acting on the motor (Milic et al. 2018). Furthermore, motor detachment occurs more easily under assisting loads than under hindering loads (McHugh et al. 2018) (Figure 11.2A). This implies that motors experiencing assisting forces are more prone to slip along the microtubule, while motors that experience hindering loads are more likely to grip the microtubule tightly (McHugh et al. 2018). This grip state can be further reinforced by the microtubule-associated protein (MAP) Tpx2 (targeting protein for xKlp2) (Wittmann et al. 1998, Wittmann et al. 2000), which binds to the (dimerised) leucine-zipper motif near the C-terminus of Klp2 (Wittmann et al. 1998) (aa1359-1380 in hKIF15, (Figure 11.1). In Humans, binding of TPX2 (from now on hTPX2) locks the motor even more tightly to the microtubule, allowing the motor to withstand forces (much) higher than its stall force without detaching from the microtubule (Drechsler et al. 2014, Mann, Balchand, and Wadsworth 2017) (Figure 11.2B). These hKIF15:hTPX2 complexes are likely to form in situ on the microtubule as, in solution, the affinity of hKIF15 and hTPX2 for one another is relatively low (Drechsler et al. 2014).

\subsubsection{Microtubule Interaction}

Being tetrameric, hKIF15 can cross-link and bundle microtubules via its two sets of two motor domains. However, dimeric hKIF15 also retains the capacity to cross-link microtubules via a second, nucleotide-independent microtubule-binding site in the motor domain proximal section of the stalk (aa400-700). This interaction depends on electrostatic attraction to the negatively charged tubulin C-termini exposed on the microtubule surface (Reinemann et al. 2017, Sturgill et al. 2014). The cross-linking and motor activities of recombinant full-length tetrameric hKIF15 are sufficient to sort microtubules into stable parallel bundles (Drechsler and McAinsh 2016, Reinemann et al. 2017) (Figure 11.2C). This sorting mechanism involves dynamic collectives of hKIF15 motors driving an adaptive microtubule transport/parallel sliding mechanism at microtubule intersections. While the motor domain pairs of the hKIF15 tetramer move along both microtubules which they cross-link, a velocity differential between them is evident, which gradually depends on the microtubule geometry at the intersection. On parallel microtubules, the velocity differential is small, resulting 


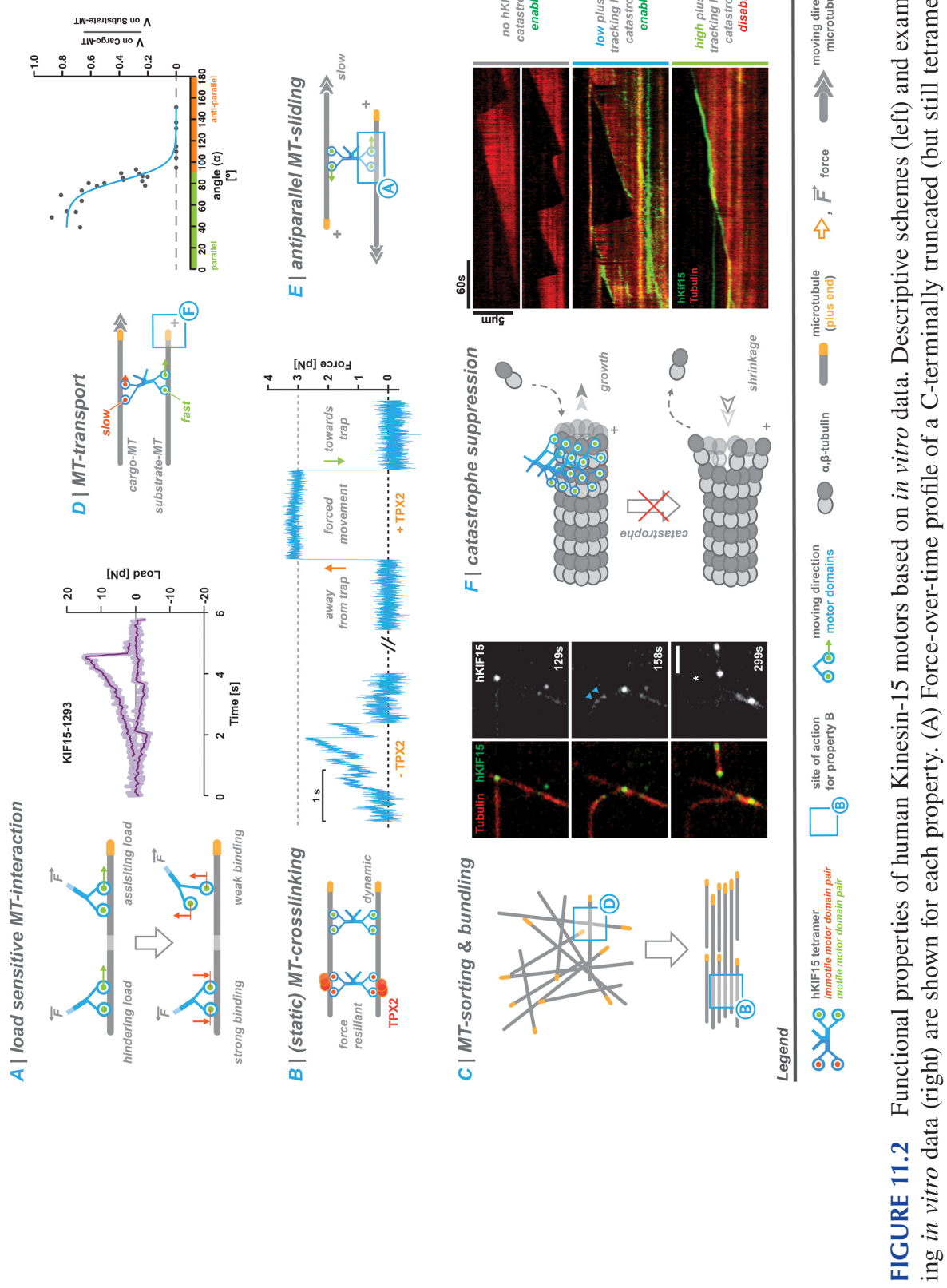




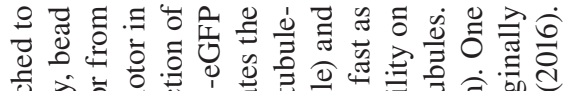

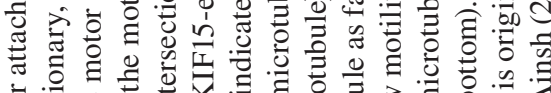

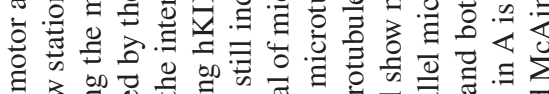

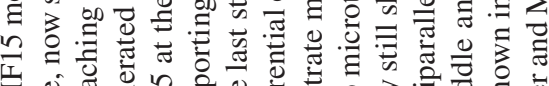

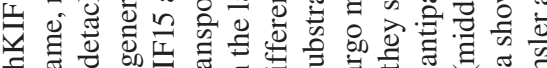

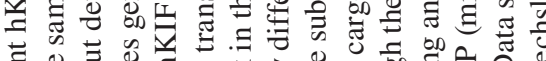

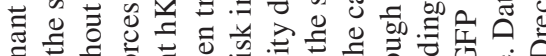

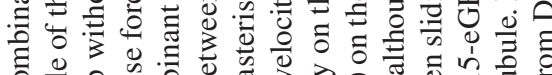

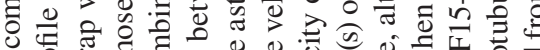
ญ。 ส

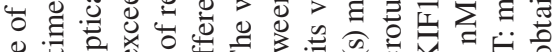

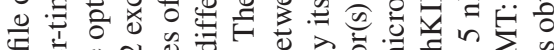

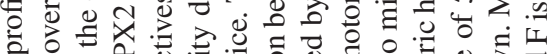

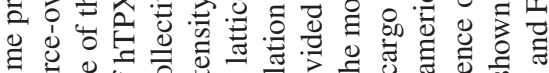

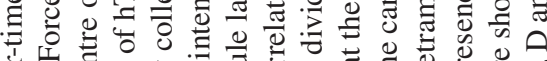

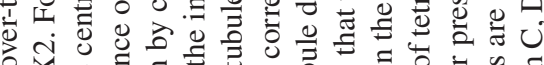
ix

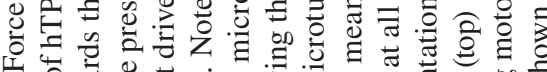

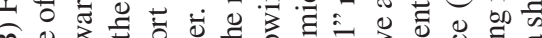
थิ

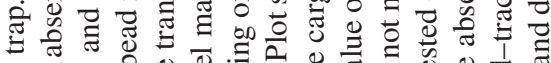

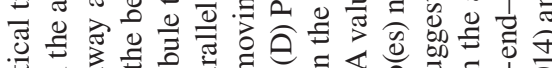

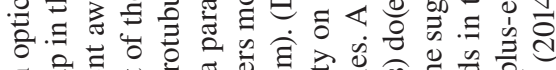

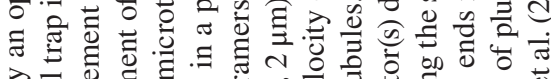

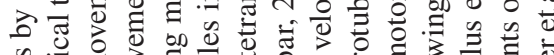

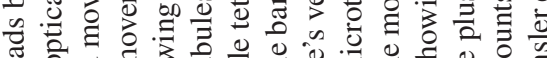

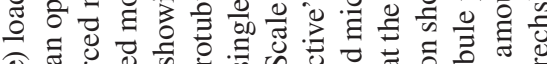
(1) 论

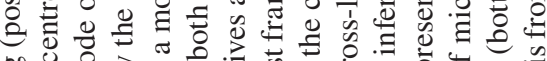

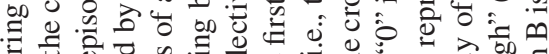

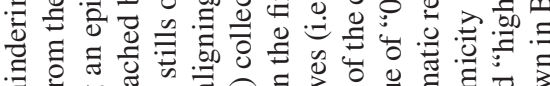

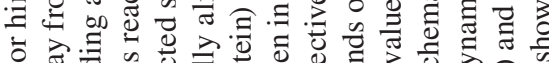

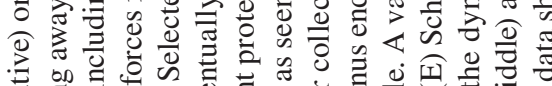
จै

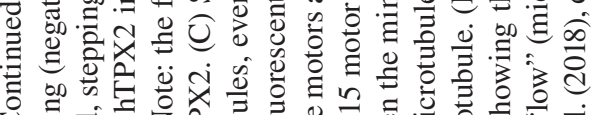

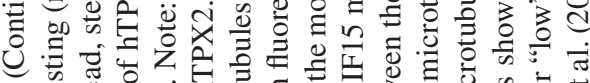

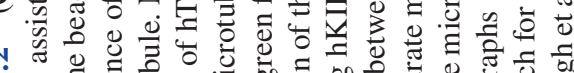

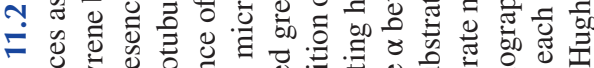

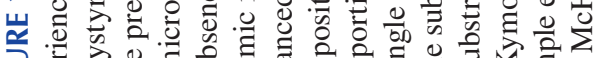

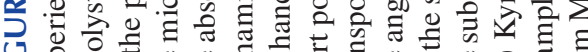

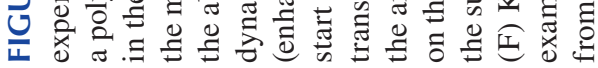


in limited parallel sliding. On antiparallel microtubules, the velocity differential is high, as only one motor domain pair is moving, transporting one microtubule along the other (Drechsler and McAinsh 2016) (Figure 11.2D). Transport velocities negatively correlate with the hKIF15 motor collective size and range between 25 and 250 $\mathrm{nm} \mathrm{s}^{-1}$ (Drechsler and McAinsh 2016). Using a force-calibrated optical trap, it was shown that dimeric hKIF15 supports the relative sliding of antiparallel microtubules (Reinemann et al. 2017) (Figure 11.2E). However, motor forces during antiparallel sliding are not cumulative across the overlap, and sliding occurs only at very low velocities $\left(<1 \mathrm{~nm} \mathrm{~s}^{-1}\right)$ (Reinemann et al. 2017) compared with the velocity of single motors in an optical-trap assay $\left(>60 \mathrm{~nm} \mathrm{~s}^{-1}\right.$ ) (Reinemann et al. 2017, Drechsler et al. 2014) or antiparallel microtubule sliding driven by Kinesin-5, which supports sliding velocities up to $50 \mathrm{~nm} \mathrm{~s}^{-1}$ (Reinemann et al. 2017, Shimamoto, Forth, and Kapoor 2015, Kapitein et al. 2005). Like other kinesins (Du, English, and Ohi 2010, Varga et al. 2009, Chen and Hancock 2015, Chen et al. 2019), hKIF15 motors additionally modulate microtubule dynamics, as plus-end-tracking hKIF15 motors suppress microtubule catastrophe events in a motor number threshold-dependent manner (Drechsler and McAinsh 2016) (Figure 11.2F).

\subsection{PHYSIOLOGICAL ROLE}

Kinesin-15 is a highly versatile motor that is implicated in multiple cellular processes, including mitotic spindle assembly/maintenance and post-mitotic processes such as cell migration, the axonal outgrowth of neurons or the production of red blood platelets from megakaryocytes (i.e., thrombopoiesis).

\subsubsection{Roles In Mitosis}

Assembly and maintenance of the mitotic spindle requires the balanced activity of three mitotic motors: Kinesin-5, Kinesin-15 and dynein (van Heesbeen, Tanenbaum, and Medema 2014). Spindle assembly starts with Kinesin-5-driven separation of centrosomes in prophase, followed by nuclear envelope breakdown, at which point microtubules from the two asters interact and self-organise the bipolar spindle. During prometaphase, Kinesin-15 associates with spindle microtubules in a hTPX2-dependent manner and can compensate for loss of Kinesin-5 function (Vanneste et al. 2009, Tanenbaum et al. 2009) (Figure 11.3, upper panels). This is because Kinesin-5 and -15 both create outward, spindle-extending forces, that are counteracted by dynein-dependent inward forces (van Heesbeen, Tanenbaum, and Medema 2014). Kinesin-5 activity is essential for early spindle pole separation, as prophase and prometaphase force production by Kinesin-15 is not sufficient to counteract dynein-dependent compressing forces (Tanenbaum et al. 2009, Vanneste et al. 2009, van Heesbeen, Tanenbaum, and Medema 2014). However, once the force equilibrium has changed - either by lowering dyneindependent forces (van Heesbeen, Tanenbaum, and Medema 2014) or by increasing Kinesin-15 activity (Sturgill and Ohi 2013, Tanenbaum et al. 2009) - Kinesin-15 is capable of driving spindle assembly even in the absence of Kinesin-5. In fact, Kinesin-15 becomes essential to spindle assembly in cells that are chronically 


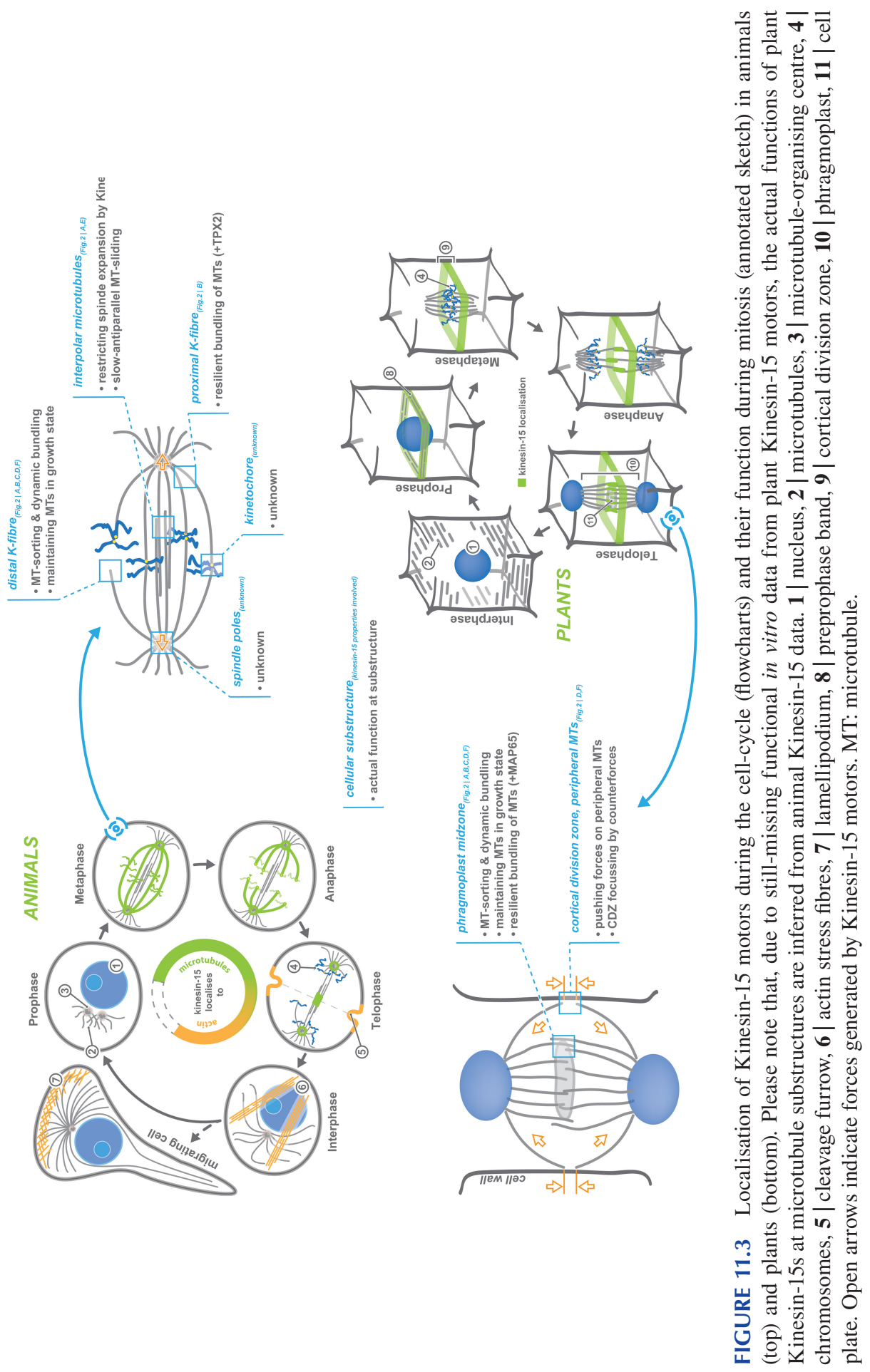


deprived of Kinesin-5 (Raaijmakers et al. 2012, Sturgill et al. 2016), and cells often increase their Kinesin-15 expression in order to maintain their capacity to divide in the absence of Kinesin-5 (Sturgill et al. 2016, Sturgill and Ohi 2013, Raaijmakers et al. 2012).

While there is a broad agreement on the mechanism by which Kinesin-5 drives spindle assembly (Kapoor 2017), the Kinesin-15 mechanism(s) remain under debate. One model proposes that Kinesin-15 - in analogy to Kinesin-5 directly drives spindle extension by sliding apart antiparallel overlaps of interpolar microtubules at the spindle midzone (Tanenbaum et al. 2009, Sturgill et al. 2014, Reinemann et al. 2017). Experimental support for this model currently appears weak as: (i) in vivo hKIF15 mainly localises to k-fibres instead of localising to antiparallel microtubule overlaps in the spindle midzone (Sturgill and Ohi 2013); (ii) hKIF15 restricts rather than supports the force generation by Kinesin-5 on interpolar microtubule overlaps (Sturgill and Ohi 2013); (iii) spindle assembly by overexpressed hKIF15 in the absence of Kinesin-5 occurs late in prometaphase by a "reverse jack-knife" mechanism (Figure 11.4) (Sturgill and Ohi 2013, Toso et al. 2009). This mechanism is fundamentally different from the axial spindle elongation driven by Kinesin-5- dependent antiparallel microtubule sliding at interpolar microtubule overlaps (Kapitein et al. 2005); and (iv) antiparallel sliding of microtubules in vitro has not been observed with tetrameric hKIF15 motors (Drechsler and McAinsh 2016), and the sliding velocities with dimeric KIF15s $\left(<1 \mathrm{~nm} \mathrm{~s}^{-1}\right)$ (Reinemann et al. 2017) are almost two orders of magnitude slower than those generated by ensembles of Kinesin-5 in a comparable setup (Shimamoto, Forth, and Kapoor 2015). As a consequence, assembly of a 10- $\mu \mathrm{m}$ spindle, via a linear sliding

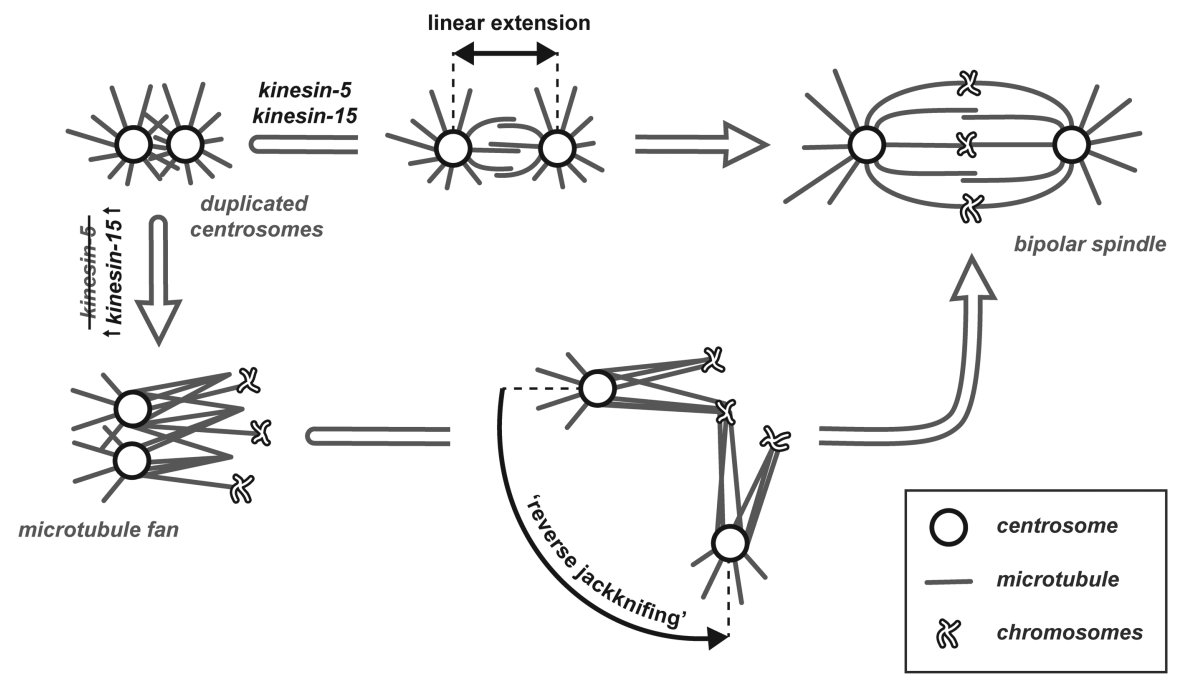

FIGURE 11.4 Scheme showing mitotic spindle assembly in the presence (top, linear extension) and absence (bottom, reverse jack-knifing) of Kinesin-5. Please note that Kinesin-15driven spindle assembly requires additional microtubule-bundling activity e.g., by Kinesin- 15 overexpression. 
mechanism, would take hours when carried out by Kinesin-15, instead of taking minutes in the case of Kinesin-5. In cells, however, the contribution of Kinesin-15 to metaphase spindle elongation appears to have a magnitude of about $1 \mu \mathrm{m} \mathrm{min} \mathrm{m}^{-1}$ (Tanenbaum et al. 2009). An alternative model suggests that Kinesin-15 localises to k-fibres (Sturgill and Ohi 2013), where it indirectly drives spindle expansion by organising and stabilising k-fibres (Brouwers, Mallol Martinez, and Vernos 2017, van Heesbeen, Tanenbaum, and Medema 2014) through stable cross-links (Drechsler et al. 2014), while also harmonising their dynamics (Drechsler and McAinsh 2016). The idea is that k-fibres continuously grow into the attached kinetochores, creating expansive forces within the spindle that lead to centrosome separation (Toso et al. 2009). Since this mechanism requires extra bundling activity, it might explain why a cell chronically deprived of Kinesin-5 activity has to either increase hKIF15 levels (Sturgill and Ohi 2013), or provide extra bundling activity in the form of a rigor Kinesin-5 to allow spindle assembly at normal hKIF15 levels (Sturgill et al. 2016).

Finally, Kinesin-15-dependent microtubule sorting and bundling, but not antiparallel sliding, is key to microtubule rearrangements in other organisms: During the acentrosomal spindle assembly of $C$. elegans meiosis, the Kinesin-15, KLP-18, sorts spindle microtubules into parallel arrays, which enables the subsequent focusing of microtubule minus ends into defined spindle poles and therefore allows the establishment of a bipolar spindle (Wolff et al. 2016, Segbert et al. 2003). Moreover, in the moss $P$. patens, the Kinesin-15 motors, KINID1a and KINID1b, dynamically focus microtubule plus ends and bundle microtubules in apical domes of caulonemal apical cells during polarised tip growth (Hiwatashi, Sato, and Doonan 2014).

During mitosis, Kinesin-15 localises to additional cellular substructures, including the spindle poles (Buster et al. 2003, Mann, Balchand, and Wadsworth 2017, Boleti, Karsenti, and Vernos 1996), the spindle midbody (Buster et al. 2003, Rogers et al. 2000) and the kinetochores (Mann, Balchand, and Wadsworth 2017). Through an interaction of amino acids 1017-1238 with the chromosomal periphery marker KI-67 (Figure 11.1 (Booth et al. 2014, Sueishi, Takagi, and Yoneda 2000, Vanneste et al. 2009)), Kinesin-15 also localises to the chromosomal periphery (Figure 11.3, upper panels (Buster et al. 2003, Mann, Balchand, and Wadsworth 2017, Vanneste et al. 2009, Brouwers, Mallol Martinez, and Vernos 2017)). Further Kinesin-15 subpopulations have been reported to localise to the cleavage furrow during cytokinesis, as well as to actin stress fibres of interphase cells (Figure 11.3, upper panels (Buster et al. 2003)). The detection of those minor Kinesin-15 populations, however, appears to depend on the experimental conditions (i.e., visualisation method, antibodies, spatial resolution; for example, compare (Mann, Balchand, and Wadsworth 2017) with (Brouwers, Mallol Martinez, and Vernos 2017) and (Sturgill and Ohi 2013). Particular subpopulations might also be organism-specific, as Kinesin-15 is present at the spindle poles in rat (Buster et al. 2003) and frog (Boleti, Karsenti, and Vernos 1996), but not in sea urchin (Rogers et al. 2000). Conversely, Kinesin-15 localises to the spindle midbody in rat (Buster et al. 2003) and sea urchin (Rogers et al. 2000), but not in the frog (Boleti, Karsenti, and Vernos 1996). The functions of Kinesin-15 at these diverse locations are currently not known and warrant further study. 


\subsubsection{Establishing the Cortical Division Zone and Phragmoplast Assembly in Plants}

In plants, the site of cell division is already set at the beginning of mitosis. From late S-phase to prometaphase, the preprophase band (PPB) - made of endomembrane, F-actin and microtubules - lines the cell cortex and marks (but not necessarily determines) the future division site (Müller 2019). Subsequently, additional factors like the microtubule-associated protein TAN (Walker et al. 2007) and the small GTPaseactivating proteins PHGAP1,2 (Stockle et al. 2016) and RANGAP1 (Xu et al. 2008) are recruited to the PPB-site in a Kinesin-15 (i.e., POK1 and POK2)-dependent manner, and persist there throughout mitosis, conserving the spatial information (now termed cortical division zone, CDZ) of the PPB after its disassembly at the end of prophase (Figure 11.3, lower panels). Targeting and tethering of POK1 and POK2 to the $\mathrm{PPB} / \mathrm{CDZ}$ is mediated by their C-termini and does not depend on their motor activity or the presence of microtubules. Additionally, the C-termini of POK1 and POK2 also mediate the long-term tethering of CDZ factors at the PPB/CDZ (Figure 11.1 (Lipka et al. 2014, Herrmann et al. 2018)).

Later, during cytokinesis, plant cells divide by the radial insertion of a cell plate into the plane marked by the CDZ (Figure 11.3, lower panels). Cell plate growth is supported by the so-called phragmoplast, two arrays of parallel microtubules (i.e., remnants of the former spindle) in between the re-forming nuclei that orient with their plus ends towards the cell division plane, forming antiparallel overlaps (Ho et al. 2011, Jurgens 2005). The dynamic phragmoplast targets vesicles containing cell-plate material to the nascent cell plate and provides structural support (van OostendeTriplet et al. 2017, Smertenko et al. 2018, Jurgens 2005). The various plant Kinesin-15 members contribute on multiple levels to the organisation of the phragmoplast. In the moss $P$. patens, the Kinesin-15s KINID1a and $1 \mathrm{~b}$ localise to the spindle midzone from metaphase onwards and organise the antiparallel microtubule overlaps in the phragmoplast midzone during cytokinesis, keeping the phragmoplast halves together (Hiwatashi et al. 2008). Similarly, AtPAKRP1 and AtPAKRP1L localise to the spindle midzone from anaphase onwards (Lee and Liu 2000, Pan, Lee, and Liu 2004) and are believed to organise the phragmoplast midzone, as AtPAKRP1 and AtPAKRP1L double mutants fail to assemble antiparallel microtubule arrays or cell plates (Lee, Li, and Liu 2007). Also, AtPOK2 (Herrmann et al. 2018), but not AtPOK1 (Lipka et al. 2014), localises to the phragmoplast midzone. AtPOK2 mutants however show no phragmoplast assembly phenotype, but show reduced phragmoplast expansion growth (within the cell division plane) during radial cell plate growth (Herrmann et al. 2018). The mechanism by which POK2 contributes to phragmoplast expansion has not been addressed in detail yet, but it has been suggested to promote microtubule growth at the midzone - in analogy to its animal counterpart (Herrmann et al. 2018). Phragmoplast targeting of Kinesin-15 depends on its motor activity, but sub-targeting to the phragmoplast midzone depends on MAP65 isoforms that sequester AtPOK2 there via dual interactions with both the $\mathrm{N}$-terminal extension and the $\mathrm{C}$-terminus of POK2 (Figure 11.1 (Herrmann et al. 2018)). Finally, AtPOK1 and AtPOK2 keep the phragmoplast perpendicular to the division plane as it is marked by the cortical division zone (Müller, Han, and Smith 2006, Herrmann et al. 2018). Consequently, 
unaligned phragmoplasts in the absence of POK activity cause random insertion of cell walls and therefore heavily disordered plant tissues (Müller, Han, and Smith 2006, Herrmann et al. 2018). Phragmoplast alignment was suggested to occur by cortical POK motors at the CDZ, that act on peripheral phragmoplast microtubules (Chugh et al. 2018, Müller 2019). By walking to their plus ends, tethered motors create pushing forces against the expanding phragmoplast, thereby aligning it perpendicular to the CDZ plane. Simultaneously, these motors experience a counterforce by the phragmoplast which focuses them - and thereby the CDZ - on the cortex, a process that has been observed in vivo during progression of cytokinesis (Figure 11.3, lower left panel (Müller 2019)). Interpretation of POK1 and POK2 knockdown data, however, might be difficult, given that the CDZ identity (i.e., the localisation of TAN/ PHGAP1,2/RANGAP1) strongly depends on POK-dependent targeting and tethering.

\subsubsection{Postmitotic Functions in Neuronal Development}

Expression of Kinesin-15 is particularly high in tissues of the vertebrate nervous system (i.e., brain, spinal cord, olfactory bulb, otic vesicle and retina) during embryonal development and early postnatal phases (Xu et al. 2014, Liu et al. 2010). Here, Kinesin-15 contributes, by as yet largely unknown mechanisms, to the control of cell morphology and cell migration. Upon depletion of Kinesin-15, neurites (i.e., dendrites and axons) are growing out faster and longer than in unperturbed control neurons, but exhibit a smaller diameter (Lin et al. 2012, Liu et al. 2010, Dong et al. 2019). This phenotype was explained by the mobilisation of short microtubule fragments in the absence of Kinesin-15 (Liu et al. 2010) and suggests that Kinesin-15 in neurons also fulfils a primarily structural, microtubule-organising function. In line with that, Kinesin-15-depleted neurons show additional microtubule-organising defects, as microtubules that orient with their plus end to the cell body are largely absent from the dendrites (Lin et al. 2012) and axons develop fewer branches and filopodia (Liu et al. 2010, Dong et al. 2019). The growth cones of Kinesin-15-depleted cells are considerably smaller than those of control cells and have lost the ability to control their growth direction (Liu et al. 2010). This phenotype might result from perturbed, Kinesin-15-dependent interactions between the microtubule and the actin cytoskeleton in the growth cone (Liu et al. 2010). In accordance, the existence of a Kinesin-15 and myosin IIB heterotetramer has been proposed, based on observations in migrating astrocytes (Feng et al. 2016). Here, disruption of the reported physical interaction between Kinesin-15 and myosin IIB (see Figure 11.1) increases astrocyte migration, phenocopying a Kinesin-15-depletion phenotype (Feng et al. 2016). In developing neurons, therefore, Kinesin-15 shows both microtubule- and actin-related activity at the same time. This stands in stark contrast to mitosis, where both functions are temporarily separated, suggesting that Kinesin-15 activities are posttranslationally fine-tuned to match the respective scenarios.

\subsection{INVOLVEMENT IN DISEASE}

A number of studies propose hKIF15 (i.e., hKIF15 overexpression) as a biomarker for a multitude of cancer types (Liu et al. 2018, Zhao et al. 2019, Song et al. 2018, Zou et al. 2014, Sheng, Jiang, and Xue 2019, Menyhart, Pongor, and Gyorffy 2019, 
Stangeland et al. 2015, Chen et al. 2017, Qiao et al. 2018, Yu et al. 2019, Wang et al. 2017), and suggest that KIF15 promotes tumour cell proliferation (Zhao et al. 2019, Zou et al. 2014, Qiao et al. 2018, Yu et al. 2019, Wang et al. 2017). However, whether hKIF15 overexpression simply reflects higher proliferative rates remains unclear and the mechanism by which hKIF15 promotes cell proliferation has not yet been established. On the other hand, there is an emerging view that hKIF15 is a valuable target for anti-cancer therapies (Rath and Kozielski 2012). While robust spindle assembly and maintenance due to the functional redundancy of Kinesins- 5 and -15 is, without doubt, beneficial to the cell, this failsafe mechanism poses a problem in current anticancer therapy strategies. Based on the observation that loss of Kinesin-5-dependent forces completely prevents spindle assembly in most systems (Hagan and Yanagida 1992, Heck et al. 1993, Kapoor et al. 2000) and the rationale that cancer cells exhibit a higher mitotic activity compared with somatic cells, some effort has been made to develop Kinesin-5 inhibitors. These drugs, however, failed in Phase II clinical trials, presumably due to the functional redundancy of Kinesin-5 and -15 (Rath and Kozielski 2012, Chandrasekaran, Tátrai, and Gergely 2015). In fact, cells that have been chronically exposed to Kinesin-5 inhibitors acquire drug resistance in a strictly Kinesin-15dependent manner (Sturgill et al. 2016, Sturgill and Ohi 2013). Hence, first approaches have been made to develop Kinesin-15 inhibitors that are aimed at inhibiting tumour growth when co-administered with established Kinesin-5 inhibitors (Sebastian 2017, Dumas et al. 2019, Milic et al. 2018). So far, two Kinesin-15 inhibitors have been described, KIF15-IN-1 and GW108X. KIF15-IN-1 is an ATP-competitive inhibitor (Dumas et al. 2019) that inhibits (C-terminally truncated) hKIF15 with a half-maximal inhibitory concentration $\left(\mathrm{IC}_{50}\right.$ ) of $0.2 \mu \mathrm{M}$ (Dumas et al. 2019) to $1.7 \mu \mathrm{M}$ (Milic et al. 2018) in vitro. GW108X is an allosteric inhibitor that inhibits (C-terminally truncated) hKIF15 with an IC $_{50}$ of $0.8 \mu \mathrm{M}$ (Dumas et al. 2019). Whereas KIF15-IN-1 is "specific" for hKIF15 (Milic et al. 2018), GW108X shows some inhibitory potential for Kinesin-5 as well (Dumas et al. 2019). Using KIF15-IN-1, it could be shown that co-administration of Kinesin-5 and -15 inhibitors inhibits the growth of cancer cells (Milic et al. 2018). Hence, these early inhibitors will be promising starting points for the development of further refined Kinesin-15 inhibitors.

A recent case study reported a mutation within the hKIF15 gene that phenocopies Braddock-Carey syndrome (BCS) (Sleiman et al. 2017). BCS is characterised by multiple developmental aberrations, mainly affecting the morphology of the head (i.e., Pierre-Robin sequence and distinctive facies) and the development of the brain (i.e., microcephaly and agenesis of the corpus callosum), as well as by a congenital thrombocytopaenia (Braddock et al. 2016). Classically, BCS is caused by microdeletions on chromosome 21 (i.e., 21q22.11), affecting at least three different contiguous genes (SON, ITSN1 and RUNX1) (Braddock et al. 2016), each of which could be linked to certain phenotypes of BCS. Such deletions affecting the haematopoietic transcription factor RUNX1 (Okuda et al. 2001, Sood, Kamikubo, and Liu 2017), are associated with the occurrence of congenital thrombocytopenia. In this light, it is even more surprising that a single mutation within the hKIF15 open reading frame, introducing a premature stop codon (R501*), can phenocopy such a complex syndrome (Sleiman et al. 2017). However, it emphasises the role of Kinesin-15 during neuronal development and points to a - so far unknown - role of Kinesin-15 in 
thrombopoiesis, a process that relies heavily on a functional actin and microtubule cytoskeleton (Favier and Raslova 2015).

\section{REFERENCES}

Acar, S., D. B. Carlson, M. S. Budamagunta, V. Yarov-Yarovoy, J. J. Correia, M. R. Ninonuevo, W. T. Jia, L. Tao, J. A. Leary, J. C. Voss, J. E. Evans, and J. M. Scholey. 2013. “The bipolar assembly domain of the mitotic motor kinesin-5." Nature Communications 4. doi:ARTN 134310.1038/ncomms2348.

Boleti, H., E. Karsenti, and I. Vernos. 1996. "Xklp2, a novel Xenopus centrosomal kinesinlike protein required for centrosome separation during mitosis." Cell 84 (1):49-59. doi:S0092-8674(00)80992-7 [pii].

Booth, D. G., M. Takagi, L. Sanchez-Pulido, E. Petfalski, G. Vargiu, K. Samejima, N. Imamoto, C. P. Ponting, D. Tollervey, W. C. Earnshaw, and P. Vagnarelli. 2014. "Ki-67 is a PP1-interacting protein that organises the mitotic chromosome periphery." Elife 3:e1641. doi:10.7554/eLife.01641.

Braddock, S. R., S. T. South, J. D. Schiffman, M. Longhurst, L. R. Rowe, and J. C. Carey. 2016. "Braddock-Carey syndrome: A 21q22 contiguous gene syndrome encompassing RUNX1." American Journal of Medical Genetics Part A 170 (10):2580-6. doi:10.1002/ajmg.a.37870.

Britto, M., A. Goulet, S. Rizvi, O. von Loeffelholz, C. A. Moores, and R. A. Cross. 2016. "Schizosaccharomyces pombe kinesin-5 switches direction using a steric blocking mechanism." Proceedings of the National Academy of Sciences of the United States of America 113 (47):E7483-9. doi:10.1073/pnas.1611581113.

Brouwers, Nathalie, Nuria Mallol Martinez, and Isabelle Vernos. 2017. "Role of Kif15 and its novel mitotic partner KBP in K-fiber dynamics and chromosome alignment." PLoS one 12 (4):e0174819. doi:10.1371/journal.pone.0174819.

Buster, Daniel W., Douglas H. Baird, Wenqian Yu, Joanna M. Solowska, Muriel Chauvière, Agnieszka Mazurek, Michel Kress, and Peter W. Baas. 2003. "Expression of the mitotic kinesin Kif15 in postmitotic neurons: Implications for neuronal migration and development." Journal of Neurocytology 32 (1):79-96. doi:10.1023/a:1027332432740.

Chandrasekaran, Gayathri, Péter Tátrai, and Fanni Gergely. 2015. "Hitting the brakes: Targeting microtubule motors in cancer." British Journal of Cancer 113:693. doi:10.1038/bjc.2015.264.

Chen, G. Y., J. M. Cleary, A. B. Asenjo, Y. Chen, J. A. Mascaro, D. F. J. Arginteanu, H. Sosa, and W. O. Hancock. 2019. "Kinesin-5 promotes microtubule nucleation and assembly by stabilizing a lattice-competent conformation of tubulin." Current Biology 29 (14):2259-69 e4. doi:10.1016/j.cub.2019.05.075.

Chen, J., S. Li, S. Zhou, S. Cao, Y. Lou, H. Shen, J. Yin, and G. Li. 2017. "Kinesin superfamily protein expression and its association with progression and prognosis in hepatocellular carcinoma." Journal of Cancer Research and Therapeutics 13 (4):651-9. doi:10.4103/ jcrt.JCRT_491_17.

Chen, Y. L., and W. O. Hancock. 2015. "Kinesin-5 is a microtubule polymerase." Nature Communications 6. doi:ARTN 816010.1038/ncomms9160.

Chugh, M., M. Reissner, M. Bugiel, E. Lipka, A. Herrmann, B. Roy, S. Muller, and E. Schaffer. 2018. "Phragmoplast orienting kinesin 2 is a weak motor switching between processive and diffusive modes." Biophysical Journal 115 (2):375-85. doi:10.1016/j.bpj.2018.06.012.

Dong, Zhangji, Shuwen Wu, Chenwen Zhu, Xueting Wang, Yuanyuan Li, Xu Chen, Dong Liu, Liang Qiang, Peter W. Baas, and Mei Liu. 2019. "Clustered Regularly Interspaced Short Palindromic Repeats (CRISPR)/Cas9-mediated kif15 mutations accelerate axonal outgrowth during neuronal development and regeneration in zebrafish." Traffic 20 (1):71-81. doi:10.1111/tra.12621. 
Drechsler, H., T. McHugh, M. R. Singleton, N. J. Carter, and A. D. McAinsh. 2014. "The Kinesin-12 Kif15 is a processive track-switching tetramer." Elife 3:e01724. doi:10.7554/ eLife.01724.

Drechsler, H., A. N. Tan, and D. Liakopoulos. 2015. "Yeast GSK-3 kinase regulates astral microtubule function through phosphorylation of the microtubule-stabilizing kinesin Kip2." Journal of Cell Science 128 (21):3910-21. doi:10.1242/jcs.166686.

Drechsler, Hauke, and Andrew D. McAinsh. 2016. "Kinesin-12 motors cooperate to suppress microtubule catastrophes and drive the formation of parallel microtubule bundles." Proceedings of the National Academy of Sciences of the United States of America 113 (12):E1635-44. doi:10.1073/pnas.1516370113.

Du, Y., C. A. English, and R. Ohi. 2010. "The kinesin-8 Kif18A dampens microtubule plusend dynamics." Current Biology 20 (4):374-80. doi:10.1016/j.cub.2009.12.049.

Dumas, Megan E., Geng-Yuan Chen, Nicole D. Kendrick, George Xu, Scott D. Larsen, Somnath Jana, Alex G. Waterson, Joshua A. Bauer, William Hancock, Gary A. Sulikowski, and Ryoma Ohi. 2019. "Dual inhibition of Kif15 by oxindole and quinazolinedione chemical probes." Bioorganic \& Medicinal Chemistry Letters 29 (2):148-54. doi:10.1016/j.bmcl.2018.12.008.

Favier, R., and H. Raslova. 2015. "Progress in understanding the diagnosis and molecular genetics of macrothrombocytopenias." British Journal of Haematology 170 (5):62639. doi:10.1111/bjh.13478.

Feng, Jie, Zunlu Hu, Haijiao Chen, Juan Hua, Ronghua Wu, Zhangji Dong, Liang Qiang, Yan Liu, Peter W. Baas, and Mei Liu. 2016. "Depletion of kinesin-12, a myosin-IIBinteracting protein, promotes migration of cortical astrocytes." Journal of Cell Science 129 (12):2438-47. doi:10.1242/jcs.181867.

Gruneberg, U., R. Neef, X. Li, E. H. Chan, R. B. Chalamalasetty, E. A. Nigg, and F. A. Barr. 2006. "KIF14 and citron kinase act together to promote efficient cytokinesis." The Journal of Cell Biology 172 (3):363-72. doi:10.1083/jcb.200511061.

Hagan, Iain, and Mitsuhiro Yanagida. 1992. "Kinesin-related cut 7 protein associates with mitotic and meiotic spindles in fission yeast." Nature 356 (6364):74-6. doi:10.1038/356074a0.

Heck, M M, A Pereira, P Pesavento, Y Yannoni, A C Spradling, and L S Goldstein. 1993. "The kinesin-like protein KLP61F is essential for mitosis in Drosophila." The Journal of Cell Biology 123 (3):665-79. doi:10.1083/jcb.123.3.665.

Herrmann, A., P. Livanos, E. Lipka, A. Gadeyne, M. T. Hauser, D. Van Damme, and S. Muller. 2018. "Dual localized kinesin-12 POK2 plays multiple roles during cell division and interacts with MAP65-3." Embo Reports 19 (9). doi:ARTN e4608510.15252/ embr.201846085.

Hiwatashi, Y., M. Obara, Y. Sato, T. Fujita, T. Murata, and M. Hasebe. 2008. "Kinesins are indispensable for interdigitation of phragmoplast microtubules in the moss Physcomitrella patens.” Plant Cell 20 (11):3094-106. doi:10.1105/tpc.108.061705.

Hiwatashi, Y., Y. Sato, and J. H. Doonan. 2014. "Kinesins have a dual function in organizing microtubules during both tip growth and cytokinesis in physcomitrella patens." Plant Cell 26 (3):1256-66. doi:10.1105/tpc.113.121723.

Ho, C. M. K., T. Hotta, F. L. Guo, R. Roberson, Y. R. J. Lee, and B. Liu. 2011. "Interaction of antiparallel microtubules in the phragmoplast is mediated by the microtubuleassociated protein MAP65-3 in Arabidopsis.” Plant Cell 23 (8):2909-23. doi:10.1105/ tpc.110.078204.

Hwang, Wonmuk, Matthew J. Lang, and Martin Karplus. 2008. "Force generation in kinesin hinges on cover-neck bundle formation." Structure 16 (1):62-71. doi:10.1016/j. str.2007.11.008.

Jurgens, G. 2005. “Cytokinesis in higher plants.” Annual Review of Plant Biology 56:281-99. doi:10.1146/annurev.arplant.55.031903.141636. 
Kapitein, L. C., E. J. G. Peterman, B. H. Kwok, J. H. Kim, T. M. Kapoor, and C. F. Schmidt. 2005. "The bipolar mitotic kinesin Eg5 moves on both microtubules that it crosslinks." Nature 435 (7038):114-8. doi:10.1038/nature03503.

Kapoor, Tarun M. 2017. "Metaphase spindle assembly." Biology 6 (1):8.

Kapoor, Tarun M., Thomas U. Mayer, Margaret L. Coughlin, and Timothy J. Mitchison. 2000. "Probing spindle assembly mechanisms with monastrol, a small molecule inhibitor of the mitotic kinesin, Eg5.' The Journal of Cell Biology 150 (5):975-88. doi:10.1083/jcb.150.5.975.

Khalil, Ahmad S., David C. Appleyard, Anna K. Labno, Adrien Georges, Martin Karplus, Angela M. Belcher, Wonmuk Hwang, and Matthew J. Lang. 2008. "Kinesin's coverneck bundle folds forward to generate force." Proceedings of the National Academy of Sciences 105 (49):19247-52. doi:10.1073/pnas.0805147105.

Klejnot, M., A. Falnikar, V. Ulaganathan, R. A. Cross, P. W. Baas, and F. Kozielski. 2014. "The crystal structure and biochemical characterization of Kif15: A bifunctional molecular motor involved in bipolar spindle formation and neuronal development." Acta Crystallographica Section D: Biological Crystallography 70 (Pt 1):123-33. doi:10.1107/S1399004713028721.

Lee, Y. R., Y. Li, and B. Liu. 2007. "Two Arabidopsis phragmoplast-associated kinesins play a critical role in cytokinesis during male gametogenesis." Plant Cell 19 (8):2595-605. doi:10.1105/tpc.107.050716.

Lee, Y. R., and B. Liu. 2000. "Identification of a phragmoplast-associated kinesin-related protein in higher plants." Current Biology 10 (13):797-800.

Lin, Shen, Mei Liu, Olga I. Mozgova, Wenqian Yu, and Peter W. Baas. 2012. "Mitotic motors coregulate microtubule patterns in axons and dendrites." The Journal of Neuroscience 32 (40):14033-49. doi:10.1523/jneurosci.3070-12.2012.

Lipka, Elisabeth, Astrid Gadeyne, Dorothee Stöckle, Steffi Zimmermann, Geert De Jaeger, David W. Ehrhardt, Viktor Kirik, Daniel Van Damme, and Sabine Müller. 2014. "The phragmoplast-orienting kinesin-12 class proteins translate the positional information of the preprophase band to establish the cortical division zone in Arabidopsis thaliana." Plant Cell 26 (6):2617-32. doi:10.1105/tpc.114.124933.

Liu, M., V. C. Nadar, F. Kozielski, M. Kozlowska, W. Yu, and P. W. Baas. 2010. "Kinesin-12, a mitotic microtubule-associated motor protein, impacts axonal growth, navigation, and branching." The Journal of Neuroscience 30 (44):14896-906. doi:10.1523/ JNEUROSCI.3739-10.2010.

Liu, M., Y. L. Qiu, T. Yin, Y. Zhou, Z. Y. Mao, and Y. J. Zhang. 2018. "Meta-analysis of microarray datasets identify several chromosome segregation- related cancer/testis genes potentially contributing to anaplastic thyroid carcinoma." PeerJ 6. doi:ARTN e582210.7717/peerj.5822.

Mann, B. J., S. K. Balchand, and P. Wadsworth. 2017. "Regulation of Kif15 localization and motility by the C-terminus of TPX2 and microtubule dynamics." Molecular Biology of the Cell 28 (1):65-75. doi:10.1091/mbc.E16-06-0476.

McHugh, T., H. Drechsler, A. D. McAinsh, N. J. Carter, and R. A. Cross. 2018. "Kif15 functions as an active mechanical ratchet." Molecular Biology of the Cell 29 (14):1743-52. doi:10.1091/mbc.E18-03-0151.

Menyhart, O., L. S. Pongor, and B. Gyorffy. 2019. "Mutations defining patient cohorts with elevated PD-L1 expression in gastric cancer." Frontiers in Pharmacology 9. doi:ARTN 152210.3389/fphar.2018.01522.

Milic, Bojan, Anirban Chakraborty, Kyuho Han, Michael C. Bassik, and Steven M. Block. 2018. "KIF15 nanomechanics and kinesin inhibitors, with implications for cancer chemotherapeutics." Proceedings of the National Academy of Sciences 115 (20):E4613-E4622. doi:10.1073/pnas.1801242115.

Müller, Sabine. 2019. "Plant cell division — defining and finding the sweet spot for cell plate insertion." Current Opinion in Cell Biology 60:9-18. doi:10.1016/j.ceb.2019.03.006. 
Müller, Sabine, Shengcheng Han, and Laurie G. Smith. 2006. "Two kinesins are involved in the apatial control of cytokinesis in Arabidopsis thaliana." Current Biology 16 (9):88894. doi:10.1016/j.cub.2006.03.034.

Okuda, T., M. Nishimura, M. Nakao, and Y. Fujita. 2001. "RUNX1/AML1: A central player in hematopoiesis." International Journal of Hematology 74 (3):252-7.

Pan, R., Y. R. Lee, and B. Liu. 2004. "Localization of two homologous Arabidopsis kinesin-related proteins in the phragmoplast." Planta 220 (1):156-64. doi:10.1007/ s00425-004-1324-4.

Qiao, Y., J. Chen, C. Ma, Y. Liu, P. Li, Y. Wang, L. Hou, and Z. Liu. 2018. "Increased KIF15 expression predicts a poor prognosis in patients with lung adenocarcinoma." Cellular Physiology and Biochemistry 51 (1):1-10. doi:10.1159/000495155.

Raaijmakers, J. A., R. G. van Heesbeen, J. L. Meaders, E. F. Geers, B. Fernandez-Garcia, R. H. Medema, and M. E. Tanenbaum. 2012. "Nuclear envelope-associated dynein drives prophase centrosome separation and enables Eg5-independent bipolar spindle formation." The EMBO Journal 31 (21):4179-90. doi:10.1038/emboj.2012.272.

Rath, O., and F. Kozielski. 2012. "Kinesins and cancer." Nature Reviews Cancer 12 (8):52739. doi:10.1038/nrc3310.

Reinemann, Dana N., Emma G. Sturgill, Dibyendu Kumar Das, Miriam Steiner Degen, Zsuzsanna Vörös, Wonmuk Hwang, Ryoma Ohi, and Matthew J. Lang. 2017. "Collective force regulation in anti-parallel microtubule gliding by dimeric Kif15 kinesin motors." Current Biology 27 (18):2810-20.e6. doi:10.1016/j.cub.2017.08.018.

Roberts, A. J., B. S. Goodman, and S. L. Reck-Peterson. 2014. "Reconstitution of dynein transport to the microtubule plus end by kinesin.” Elife 3:e2641. doi:10.7554/eLife.02641.

Rogers, G. C., K. K. Chui, E. W. Lee, K. P. Wedaman, D. J. Sharp, G. Holland, R. L. Morris, and J. M. Scholey. 2000. "A kinesin-related protein, KRP(180), positions prometaphase spindle poles during early sea urchin embryonic cell division." Journal of Cell Biology 150 (3):499-512.

Scholey, J. E., S. Nithianantham, J. M. Scholey, and J. Al-Bassam. 2014. "Structural basis for the assembly of the mitotic motor Kinesin-5 into bipolar tetramers." Elife 3. doi:ARTN e0221710.7554/eLife.02217.

Sebastian, J. 2017. "Dihydropyrazole and dihydropyrrole structures based design of Kif15 inhibitors as novel therapeutic agents for cancer." Computational Biology and Chemistry 68:164-74. doi:10.1016/j.compbiolchem.2017.03.006.

Segbert, Christoph, Rosemarie Barkus, Jim Powers, Susan Strome, William M. Saxton, and Olaf Bossinger. 2003. "KLP-18, a Klp2 kinesin, is required for assembly of acentrosomal meiotic spindles in Caenorhabditis elegans." Molecular Biology of the Cell 14 (11):4458-69. doi:10.1091/mbc.E03-05-0283.

Sheng, J., K. Jiang, and X. Xue. 2019. "Knockdown of Kinase family 15 inhibits cancer cell proliferation in vitro and its Clinical relevance in Triple-Negative Breast Cancer." Current Molecular Medicine. doi:10.2174/1566524019666190308122108.

Shimamoto, Y., S. Forth, and T. M. Kapoor. 2015. "Measuring pushing and braking forces generated by ensembles of kinesin- 5 crosslinking two microtubules." Developmental Cell 34 (6):669-81. doi:10.1016/j.devcel.2015.08.017.

Sleiman, Patrick M. A., Michael March, Kenny Nguyen, Lifeng Tian, Renata Pellegrino, Cuiping Hou, Walid Dridi, Mohamed Sager, Yousef H. Housawi, and Hakon Hakonarson. 2017. "Loss-of-function mutations in KIF15 underlying a BraddockCarey genocopy." Human Mutation 38 (5):507-10. doi:10.1002/humu.23188.

Smertenko, A., S. L. Hewitt, C. N. Jacques, R. Kacprzyk, Y. Liu, M. J. Marcec, L. Moyo, A. Ogden, H. M. Oung, S. Schmidt, and E. A. Serrano-Romero. 2018. "Phragmoplast microtubule dynamics - a game of zones." Journal of Cell Science 131 (2). doi:10.1242/ jcs.203331. 
Song, X., T. Zhang, X. Wang, X. Liao, C. Han, C. Yang, K. Su, W. Cao, Y. Gong, Z. Chen, Q. Han, and J. Li. 2018. "Distinct diagnostic and prognostic values of kinesin family member genes expression in patients with breast cancer." Medical Science Monitor 24:9442-64. doi:10.12659/MSM.913401.

Sood, R., Y. Kamikubo, and P. Liu. 2017. "Role of RUNX1 in hematological malignancies." Blood 129 (15):2070-82. doi:10.1182/blood-2016-10-687830.

Stangeland, Biljana, Awais A. Mughal, Zanina Grieg, Cecilie Jonsgar Sandberg, Mrinal Joel, Ståle Nygård, Torstein Meling, Wayne Murrell, Einar O. Vik Mo, and Iver A. Langmoen. 2015. "Combined expressional analysis, bioinformatics and targeted proteomics identify new potential therapeutic targets in glioblastoma stem cells." Oncotarget 6:26192-215. doi:10.18632/oncotarget.4613.

Stock, M. F., J. Chu, and D. D. Hackney. 2003. "The kinesin family member BimC contains a second microtubule binding region attached to the N terminus of the motor domain." Journal of Biological Chemistry 278 (52):52315-22. doi:10.1074/jbc.M309419200.

Stockle, D., A. Herrmann, E. Lipka, T. Lauster, R. Gavidia, S. Zimmermann, and S. Muller. 2016. "Putative RopGAPs impact division plane selection and interact with kinesin-12 POK1." Nature Plants 2 (9). doi:Artn 1612010.1038/Nplants.2016.120.

Sturgill, E. G., and R. Ohi. 2013. "Kinesin-12 differentially affects spindle assembly depending on its microtubule substrate." Current Biology 23 (14):1280-90. doi:10.1016/j. cub.2013.05.043.

Sturgill, Emma G, Dibyendu Kumar Das, Yoshimasa Takizawa, Yongdae Shin, Scott E Collier, Melanie D Ohi, Wonmuk Hwang, Matthew J Lang, and Ryoma Ohi. 2014. "Kinesin-12 Kif15 targets kinetochore fibers through an intrinsic two-step mechanism." Current Biology 24 (19):2307-13. doi:10.1016/j.cub.2014.08.022.

Sturgill, Emma G., Stephen R. Norris, Yan Guo, and Ryoma Ohi. 2016. "Kinesin-5 inhibitor resistance is driven by kinesin-12." Journal of Cell Biology 213 (2):213-27. doi:10.1083/ jcb.201507036.

Sueishi, M., M. Takagi, and Y. Yoneda. 2000. "The forkhead-associated domain of Ki-67 antigen interacts with the novel kinesin-like protein Hklp2." Journal of Biological Chemistry 275 (37):28888-92. doi:10.1074/jbc.M003879200.

Tanenbaum, M. E., L. Macurek, A. Janssen, E. F. Geers, M. Alvarez-Fernandez, and R. H. Medema. 2009. "Kif15 cooperates with eg5 to promote bipolar spindle assembly." Current Biology 19 (20):1703-11. doi:10.1016/j.cub.2009.08.027.

Toso, A., J. R. Winter, A. J. Garrod, A. C. Amaro, P. Meraldi, and A. D. McAinsh. 2009. "Kinetochore-generated pushing forces separate centrosomes during bipolar spindle assembly." Journal of Cell Biology 184 (3):365-72. doi:10.1083/jcb.200809055.

van Heesbeen, Roy G H. P., Marvin E Tanenbaum, and Rene H Medema. 2014. "Balanced activity of three mitotic motors is required for bipolar spindle assembly and chromosome segregation.” Cell Reports 8 (4):948-56. doi:10.1016/j.celrep.2014.07.015.

van Oostende-Triplet, C., D. Guillet, T. Triplet, E. Pandzic, P. W. Wiseman, and A. Geitmann. 2017. "Vesicle dynamics during plant cell cytokinesis reveals distinct developmental phases." Plant Physiology 174 (3):1544-58. doi:10.1104/pp.17.00343.

Vanneste, D., M. Takagi, N. Imamoto, and I. Vernos. 2009. "The role of Hklp2 in the stabilization and maintenance of spindle bipolarity." Current Biology 19 (20):1712-7. doi:10.1016/j.cub.2009.09.019.

Varga, V., C. Leduc, V. Bormuth, S. Diez, and J. Howard. 2009. "Kinesin-8 motors act cooperatively to mediate length-dependent microtubule depolymerization." Cell 138 (6):1174-83. doi:10.1016/j.cell.2009.07.032.

Walker, K. L., S. Mueller, D. Moss, D. W. Ehrhardt, and L. G. Smith. 2007. "Arabidopsis TANGLED identifies the division plane throughout mitosis and cytokinesis." Current Biology 17 (21):1827-36. doi:10.1016/j.cub.2007.09.063. 
Wang, Jie, Xingjun Guo, Chencheng Xie, and Jianxin Jiang. 2017. "KIF15 promotes pancreatic cancer proliferation via the MEK-ERK signalling pathway." British Journal of Cancer 117 (2):245-55. doi:10.1038/bjc.2017.165.

Wittmann, T., H. Boleti, C. Antony, E. Karsenti, and I. Vernos. 1998. "Localization of the kinesin-like protein Xklp2 to spindle poles requires a leucine zipper, a microtubuleassociated protein, and dynein." Journal of Cell Biology 143 (3):673-85.

Wittmann, Torsten, Matthias Wilm, Eric Karsenti, and Isabelle Vernos. 2000. "Tpx2, a novel Xenopus map involved in spindle pole organization.” The Journal of Cell Biology 149 (7):1405-18. doi:10.1083/jcb.149.7.1405.

Wolff, Ian D., Michael V. Tran, Timothy J. Mullen, Anne M. Villeneuve, and Sarah M. Wignall. 2016. "Assembly of Caenorhabditis elegans acentrosomal spindles occurs without evident microtubule-organizing centers and requires microtubule sorting by KLP-18/kinesin-12 and MESP-1." Molecular Biology of the Cell 27 (20):3122-31. doi:10.1091/mbc.e16-05-0291.

Xu, M., D. Liu, Z. Dong, X. Wang, Y. Liu, P. W. Baas, and M. Liu. 2014. "Kinesin-12 influences axonal growth during zebrafish neural development." Cytoskeleton (Hoboken). doi:10.1002/cm.21193.

Xu, X. F. M., Q. Zhao, T. Rodrigo-Peiris, J. Brkljacic, C. S. He, S. Muller, and I. Meier. 2008. "RanGAP1 is a continuous marker of the Arabidopsis cell division plane." Proceedings of the National Academy of Sciences of the United States of America 105 (47):1863742. doi:10.1073/pnas.0806157105.

Yu, X., X. He, L. M. Heindl, X. Song, J. Fan, and R. Jia. 2019. "KIF15 plays a role in promoting the tumorigenicity of melanoma." Experimental Eye Research. doi:10.1016/j. exer.2019.02.014.

Zhao, H. D., Q. Y. Bo, Z. L. Wu, Q. G. Liu, Y. Li, N. Zhang, H. Guo, and B. K. Shi. 2019. "KIF15 promotes bladder cancer proliferation via the MEK-ERK signaling pathway." Cancer Management and Research 11:1857-68. doi:10.2147/Cmar.S191681.

Zou, J. X., Z. Duan, J. Wang, A. Sokolov, J. Xu, C. Z. Chen, J. J. Li, and H. W. Chen. 2014. "Kinesin family deregulation coordinated by bromodomain protein ANCCA and histone methyltransferase MLL for breast cancer cell growth, survival, and tamoxifen resistance." Molecular Cancer Research 12 (4):539-49. doi:10.1158/1541-7786. MCR-13-0459. 